\title{
STUDENTS NEEDING ADDITIONAL CURRICULAR SUPPORT AND PSYCHOLOGICAL SUPPORT (SNACS, SNAPS) - A PERSPECTIVE SHIFT
}

Shivasakthy Manivasakan', K. R. Sethuraman²

\author{
${ }^{\prime}$ Reader, Dept. of Prosthodontics, \\ Indira Gandhi Institute of Dental Sciences \\ 2 Vice Chancellor, Sri Balaii Vidyapeeth
}

Sri Balaii Vidyapeeth - Mahatma Gandhi Medical College and Research Institute Campus Pillaiyarkuppam, Puducherry - 607403, India.

ABSTRACT

Labelling the students as problem learners or slow learners remains pejorative and has detrimental effects on both the students' as well as the teacher's attitude. Even though labelling is bad, a term is needed for identifying the students to enable specific support strategies that exhibit positivity. Hence better terminology of addressing the students as Students Needing Additional Curricular Support and Students Needing Additional Psychological Support are introduced. The theories behind the labelling effect on students are explained. The frameworks for identifying the student with problem, the rationale for support and the support strategies are reviewed.

\section{SNACS, SNAPS - THE TERMINOLOGY}

The students who learn slow and achieve low are termed as Students Needing Additional Curricular Support (SNACS) and Students Needing Additional Psychological Support (SNAPS) to avoid using a pejorative term like slow learners, underachievers or problem learners. ${ }^{1}$ What is the need for concern in terminology? For the reason that, the terminology here becomes a label.

The classic labelling theory by Howard Becker, states that both the concerned individual and the society are affected by the Label. The individual starts reflecting the labelled behaviour and the labeller and the society becomes prejudiced about their deeds. Often the labelled group are considered to be unacceptable. ${ }^{2,3}$

Nevertheless, to offer extra support and guidance, the group needs to be identified. Kaufmann in the context of special education had acknowledged few advantages of labelling such as easy communication between the teachers on their need, to initiate research on best practices targeting that group and to apply the evidence at a later stage for their progress. ${ }^{4}$

To gain the benefits and to avoid the detrimental effects of labelling and to develop a positive interpersonal connection between the teachers and the students ${ }^{5,6}$ based on the motivation model by Cornell, the group should be addressed in a better term. Hence the terms Students Needing Additional Curricular Support (SNACS) and Students Needing Additional Psychological Support (SNAPS) serves the purpose better.

\section{WHOM TO SUPPORT? - THE IDENTIFICATION}

Students belonging to this group have multi-faceted aetiology. Often the problem is not restricted to direct learning problem for SNACS or frank psychiatric problem for SNAPS. Vaughn et al have classified the problem learners based on the cause into four classes namely cognitive, structural, affective and interpersonal. The students in cognitive class found 
to have less knowledge, problem in written and verbal communication, spatial perception problems and lack of integration. The students in the structural class have problem in organization, time management and study habits. The affective class students have problem in memory and motivation. Many of them would have some personal life issues or health problems that lead to depression. The interpersonal class have problem in interacting with others including the teachers. The background reason might range from ethnicity issues to personal habits like substance abuse. Some may be of shy nature. ${ }^{7}$

David C. Yao et al reported insufficient clinical skills, medical knowledge and time management as problem of the medical residents, often the cause being underlying depression. ${ }^{8}$ Evans et al also reported that the students with learning difficulty exhibited deficiency in attendance, clinical skills, knowledge, communication skills and presence of anxiety. ${ }^{9}$

The identification of the students who need support plays a key role in planning effective management strategies. Moreover since learning alone is not a problem, Rachel et al stressed on differential diagnosis of the students based on the cause into three categories namely the knowledge base deficits, problems with synthesis and professionalism issues. ${ }^{10}$

Steinert proposed a framework to identify the learner's problem. The framework reflects the problems in all three domains namely the knowledge, attitude and skills. It also helps to identify whether the problem is due to the student or the teacher or the system itself. Whenever the teacher gets an intuition of something is wrong with a student, the framework can be used to identify the problem and act accordingly. ${ }^{11}$

\section{WHY TO SUPPORT? - THE RATIONALE}

Before heading on to further management, there should be a decision on to whom the problem belongs to. Apart from the learners issues discussed before, the other issues also can predispose them to fall in that group. The contributing factor could be the teacher themselves when there is a personality conflict with the student or when the expectations are far high or being judgemental. The contributing factor could be the system when the teaching is not standardized, ineffective feedback mechanism, excessive workload or unclear standards. Factors associated with the teachers and system should also be corrected respectively. ${ }^{12}$
The Self-fulfilling prophecy termed as "Pygmalion effect" by Rosenthal and Jacobson states that the students lived upto their teacher's expectations. It is further explained by a vicious cycle where a person's expectation on some other person's behaviour serves as a self-fulfilling prophecy that reinforces their self- beliefs and modify their actions accordingly which in turn increases positive expectation from the previous one. ${ }^{13}$ Therefore, additional support to the students based on their need as either towards curriculum or psychology would have a positive effect on those students.

Since the millennial generation students always desire to have positive feedback, mutual trust is needed between the student and the teacher for the feedback to be effective. Deficiency of faculty development programs and inappropriate measures of students' success often lead to teacher centred feedback which turns out to be ineffective. Unconscious incompetency is more damaging to medical students. More damaging is a direct negative feedback to these students who perceive it as personality damage. The ego block the feedback approaching the concerned in right way and hence the feedback should always be constructive. ${ }^{14}$

Hunt et al studied the frequency of occurrence of problem medical students in clinics. The students were discussed in four groups based on the frequency of occurrence and the difficulty in managing them. More frequent and difficult to manage (type1) were the students who were shy and had interpersonal problems rather than cognitive. The next frequent but not difficult to manage (type2) were those with poor knowledge and skills and inefficient time management. The less frequent and difficult to handle (type 3) were students under substance/ alcohol abuse, psychological problems and manipulative students. Infrequent and not so difficult (type 4) were those students who show hostility or unavailable at all. ${ }^{15,16}$ To reduce the stress for the students in all these categories and to prevent attrition in medical and dental schools, sufficient and constant support from the teachers is mandatory.

\section{HOW TO SUPPORT? - THE METHODS}

The support strategies begin with proper identification, defining of the problem, differentiating the contributing factor as student themselves, teacher or the system followed by the supporting strategies. ${ }^{17,12}$ Documentation needs to be done at each step. Discussion with the students on how they perceive the issue will give a detailed input for the appropriate intervention. Though certain compromise are needed 
as a part of the supporting mechanism, compromise need not be done in vital areas like the clinical posting schedule or basic expected competency required of a health profession graduate. ${ }^{17}$ Multiple interventions were discussed by Steinert et al that includes decreasing the workload of the students, change of posting batch / supervisor, availability of teachers other than class hours that facilitate individual discussions, support from mentors and peers, academic guidance, counselling and structured remedial programs. ${ }^{12}$

Vaughn et al proposed a modified S-T-P model originally given by Schmuck and Runkel's for problem solving. This model was proposed since it had similarities with the doctor patient relationship from diagnosis to treatment and could be easy for them to implement in handling the student problems too. The S-T-P model stands for Specify the problem Target state - Procedure plan. The first step (Specify the problem) is an information gathering stage which involves not only identifying the problem learner but also specifying them in which class they belong to like cognitive, interpersonal etc. The second stage (Target) involves a brainstorming session by the concerned student and the teacher on setting goals together to improve the situation. The identification does not stop with the teacher but also includes the self-assessment by the student and mutual feedback between the both. The third stage (Procedure/Plan/Path) involves decision making by the teacher on the steps to be taken. If the situation cannot be handled by the teacher's supporting strategies, then decision should be made for referral to consultants in adult learning or psychologists based on the need. Irrespective of the modality a definite follow up protocol with feedbacks is mandatory. ${ }^{7}$

The key to success of the supporting mechanisms lies in the degree of motivation experienced by the students. There is no scope for motivation unless the basic needs of the students are taken care as per the Maslow's Hierarchy of needs. ${ }^{18,19}$ Working through the steps in hierarchical needs helps to target the internal motivation and self-actualisation itself since extrinsic motivation can wean off anytime unless internalised. Setting reasonable goals in consensus with the student is one of the best supporting mechanism, as the achievement serves as a major motivation factor. The achievement motivation theory by McClelland supports the above said factors as reflectors of high achievement need. ${ }^{20}$

Motivation could be incorporated into the course by defining the course goals to the students, make them realize the relevance of the course, teaching them independent learning skills along with frequent feedback..$^{21}$ As per McGregor theory of assumptions ${ }^{22}$, theory $\mathrm{Y}$ where the teachers require an optimistic attitude on student's progress and trust is required for the supporting mechanisms to be beneficiary. Mayer et al described the emotional intelligence as an ability to perceive the emotions in correct sense which enhances the emotional growth and intellectual growth as well. ${ }^{23}$ The concept of Social and Emotional Learning (SEL) would be of benefit to the students needing the support in general to modify their thought process and inculcate positive wellbeing. The CASEL (Collaborative for Academic, Social and Emotional Learning) describes five core competencies for SEL. This includes Self-awareness, Self-management, Social awareness, Relationship skills and Responsible decision making. These skills support the students to regulate their emotions effectively that enhances their quality of interpersonal skills. The emotional and social wellbeing leads to better performance. ${ }^{24}$

\section{SUMMARY}

Shifting of the perspective towards the Students Needing Additional curricular and Psychological support (SNACS, SNAPS) is a need of the hour. Labelling them as a problematic student will further damage the situation and needs to be avoided. The identification of the problem and classifying them based on the underlying cause and determining the contributing factors are essential before starting the supporting strategies. Not only a perspective shift but a paradigm shift in the thought process and actions of the teachers are necessary for the program to be successful. The system should also make sure that there are adequate faculty development programs for the teachers in this regard and availability of multiple supporting strategies to the students' progress. 
1. Shivasakthy M, Sethuraman KR, Usha C. Learn slow, achieve low - Students Needing Additional Curricular Support and Psychological Support (SNACS, SNAPS). IERJ 2016;2(9):9-10.

2. Howard S. Becker. Outsiders. Studies in the sociology of deviance. The Free Press. Newyork. 1963.

3. Moniqueka E. Gold, Heraldo Richards. Label or Not to Label: The Special Education Question for African Americans. Educational Foundations, Winter-Spring 2012. 143-156.

4. Hallahan, D. P, \& Kauffman, J. M. Exceptional children: Introduction to special education. Englewood Cliffs, NJ: Prentice Hall.1982

5. Jonathan Muema Mwania, James Matee Muola. Teachers' Labeling of students and its effect on Students' Self- concept: A Case of Mwala District, Machakos County, Kenya. International Journal of Education and Research 2013;1(10):1-7.

6. Ercole, Jacqueline, "Labeling in the Classroom: Teacher Expectations and their Effects on Students' Academic Potential" (2009). Honors Scholar Theses. 98. http://digitalcommons.uconn.edu/srhonors_theses/98

7. Lisa M. Vaughn, Raymond C. Baker, and Thomas G. DeWitt. The Problem Learner. Teaching and Learning in Medicine 2015;10(4):217-222.

8. David C Yao, Scott M Wright. National survey of internal medicine residency program directors regarding problem residents. JAMA 2000;284(9):1099-1104.

9. Evans DE, Alstead EM, Brown J. 2010. Applying your clinical skills to students and trainees in academic difficulty. Clin Teach 7(4):230-235.

10. Rachel Bonnema, Abby Spencer, Mary Brown, Reed VanDeusen, Melissa McNeil. Dealing with the Problem Learner: learning is not always the problem! SGIM 2008, Annual Meeting April, 2008.

11. Steinert $Y$. The 'problem' junior: Whose problem is it? BMJ 2008;336(7636):150-153.

12. Yvonne Steinert. The "problem" learner: Whose problem is it? AMEE Guide No. 76 Medical Teacher 2013;35(4): e1035-e1045, D0l: 10.3109/0142159X.2013.774082.

13. Robert Rosenthal and Lenore Jacobson. Pygmalion in the classroom. The Urban Review 1968;16-20.

14. Robert G. Bing-You, Robert L. Trowbridge. Why Medical Educators May Be Failing at Feedback. JAMA 2009;302(12):1330-1.

15. Hunt DD, Carline JD, Tonesk X, Yergan J, Siever M, Loebel JP. Types of Problem Students Encountered by Clinical Teachers on Clerkships. Med Edu 1989;23:14-18.

16. Hunt DD, Khalid BA, ShahabudinSH, Rogayah J. The problem student on clinical rotations: A comparison of Malaysian and North American views. Med J Malaysia 1994;49(3):275-81.

17. William Edward Osmun, Jennifer Parr. The occasional teacher. Part 5: the learner in difficulty. Can J Rural Med 2011;16(4):131-2.

18. Nyameh Jerome. Application of the Maslow's hierarchy of need theory; impacts and implications on organizational culture, human resource and employee's performance. International Journal of Business and Management Invention 2013;2(3):39-45.

19. Avneet Kaur. Maslow's Need Hierarchy Theory: Applications and Criticisms. Global Journal of Management and Business Studies 2013;3(10):1061-64.

20. Pardee, Ronald L. Motivation theories of Maslow, Herzberg, McGregor and McClelland. A literature review of selected theories dealing with job satisfaction and motivation. 1990. Educational Resources Information Center (ERIC).

21. Capturing and Directing the Motivation to Learn. Speaking of Teaching Fall 1998;10(1):1-4.

22. Matthew Stewart. Theories $X$ and $Y$ revisited. Oxford Leadership Journal. Shifting the trajectory of civilization 2010;1(3):1-5.

23. Mayer D John, Peter Salovey. What is Emotional Intelligence? Emotional Development and Emotional Intelligence. Chapter 1;1-30.

24. Marc A. Brackett, Susan E. Rivers. Transforming students' lives with social and emotional learning. Handbook of emotions in education. Yale Centre for Emotional Intelligence. Yale University. 